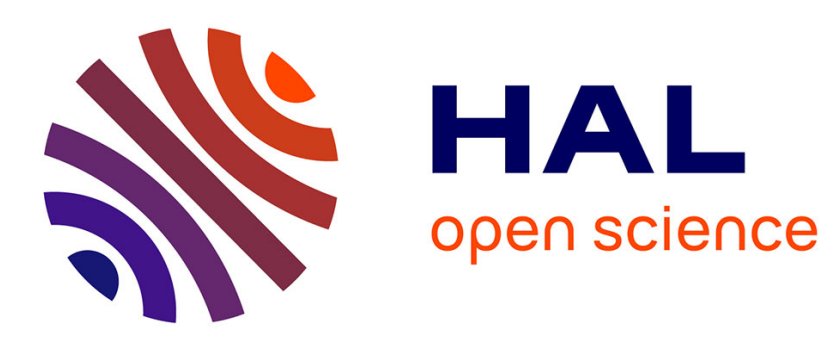

\title{
Fluid films with curvature elasticity
}

David Steigmann

\section{To cite this version:}

David Steigmann. Fluid films with curvature elasticity. Archive for Rational Mechanics and Analysis, 1999, 150 (2), pp.127-152. hal-00787206

\section{HAL Id: hal-00787206 https://hal.science/hal-00787206}

Submitted on 21 Jun 2013

HAL is a multi-disciplinary open access archive for the deposit and dissemination of scientific research documents, whether they are published or not. The documents may come from teaching and research institutions in France or abroad, or from public or private research centers.
L'archive ouverte pluridisciplinaire HAL, est destinée au dépôt et à la diffusion de documents scientifiques de niveau recherche, publiés ou non, émanant des établissements d'enseignement et de recherche français ou étrangers, des laboratoires publics ou privés. 


\title{
Fluid Films with Curvature Elasticity
}

\author{
D. J. SteigmanN
}

\section{Introduction}

The phenomenology of surfactant fluid-film microstructures interspersed in bulk fluids poses significant challenges to continuum theory. By using simple models of elastic surfaces, chemical physicists have been partially successful in describing the qualitative features of the large variety of equilibrium structures observed $[1,2]$. The basic constituent of such a surface is a polar molecule composed of hydrophilic head groups attached to hydrophobic tail groups. At low concentrations in a bulk fluid such as water, the surfactant molecules, or amphiphiles, migrate to free surfaces and arrange themselves as monomolecular films with the tail groups shielded from the bulk phase. This process is associated with a dramatic reduction of the apparent surface tension, and continues with increasing amphiphile concentration until the critical micelle concentration is reached and the molecules begin to form microstructures interspersed in the bulk [2]. These can assume a variety of forms, due to the range of mechanisms available for shielding the tail groups. The prevalent morphologies are strongly influenced by amphiphile concentration and, to a lesser degree, by temperature, but the precise factors responsible for their formation are apparently not well understood.

The basic component of these fluid microstructures is the monolayer. At fixed temperature and at amphiphile concentrations exceeding the critical value, the monolayers typically form rod-like cylinders or spherical micelles. The latter structure is also associated with surfactant-stabilized microemulsions of immiscible fluids such as water and oil, in which small droplets of one fluid are suspended in the bulk phase of the other. The interfaces separating the fluids consist of amphiphilic monolayers with the tail groups directed away from the water phase. Emulsification inversion, in which the interior and exterior phases are exchanged, is thought to be facilitated by the emergence of an intermediate bicontinuous microstructure conceived by SCRIVEN $[3,4]$. This consists of compact oriented films of high genus having a spongy or porous structure. 
Bilayer surfaces composed of oppositely oriented monolayers furnish another mechanism for shielding tail groups. These occur in a variety of forms including planar lamellae, spherical vesicles, and variants of the bicontinuous topology [2]. Bilayers of biological surfactants, called phospholipids, are also known to be of fundamental importance to the structure and function of cell walls $[5,6]$.

Typical length scales for these microstructures are so small that local interactions due to changes in the alignment of the amphiphilic molecules have a pronounced influence on surface morphology. Cosserat surface theory has been the preferred continuum model, since surfactant systems do not exist in bulk. Local effects associated with alignment are represented by assigning elastic resistance to the configurations of a director field representing molecular orientation. Variants of this model have been developed by Helfrich [7], Ericksen [8], Jenkins [9], and KRISHNASWAMY [10]. The general theory accounts for surface strain, director extension, and director tilt, the latter being associated with misalignment of the director and the surface normal. However, there is a preference in the physical-chemistry and cell-biology literatures for a simpler model based on surface geometry alone [7, 11]. This is justified by the belief that local Van der Waal's and electrostatic forces tend to act in such a way as to suppress misalignment and maintain roughly fixed tail lengths, particularly in bilayers [7, 12].

In this work I discuss a purely mechanical Cosserat theory incorporating local constraints on the director field. The film is assumed to be a material surface consisting of a fixed set of mass particles. Two essentially equivalent models are envisaged according to whether or not films are presumed to possess material symmetry. In the affirmative case an appropriate concept of fluidity is introduced based on ideas of NoLL [13] and their adaptation to material surfaces by MuRdocH \& CoHEN [14]. The relevant Legendre-Hadamard inequality for energy-minimizing equilibria is obtained and used to motivate a simple proposal for the description of bilayer response.

\section{Equilibrium of elastic films}

I use the nonlinear Kirchhoff-Love theory of shells to obtain a simple model for material fluid-film equilibria. The Kirchhoff-Love shell may be interpreted as a one-director Cosserat surface [15] with the director field constrained to coincide with the local orientation field. For amphiphilic bilayer films, the director can be thought of as a line segment formed by two hydrophobic molecular tails connecting opposed hydrophilic heads, each located at a lateral interface with the adjoining bulk fluid. The constraint is intended to represent the suppression of director tilt and extension or contraction in accordance with the generally accepted phenomenology.

The basic theory has been developed in a variety of ways. Traditionally, variational principles were used to obtain the field equations and Kirchhoff edge conditions [9, 16-19]. NAGHDI [15] advanced an alternative approach based on a set of balance, invariance, and constitutive postulates distinct from those for a Cosserat shell. I [20] recently obtained the Kirchhoff-Love theory from Naghdi's postulates for Cosserat surfaces together with local constraints on the director field and 
its gradient. The same theory may also be obtained by regarding the shell as an elastic surface with an energy per unit mass that responds to the first and second gradients of a map from a parameter plane to a surface in 3-space. The second gradient contributes both a curvature and a metric gradient. If the influence of the latter is suppressed the Kirchhoff-Love theory can be recovered via a variational argument [21].

The well-known equilibrium equations for elastic Kirchhoff-Love shells are summarized here using the notation of [20]. Thus, let $\mathbf{r}\left(\theta^{\alpha}\right)$ be the Euclidean position of a particle $x$ with convected coordinates $\theta^{\alpha} ; \alpha=1,2$, on a surface $\omega$. In this work I am mainly concerned with the local equations of the theory and therefore freely exploit the local equivalence of surfaces and parametrized surfaces. Wherever global equations are discussed it will be assumed that the relevant patch of surface either admits of a single coordinate chart or that it can be covered by the union of such patches.

Local equilibrium of forces may be expressed concisely as

$$
\mathbf{T}_{; \alpha}^{\alpha}+\rho \mathbf{f}=\mathbf{0}
$$

where $\rho$ is the mass of the film measured per unit area of $\omega, \mathbf{f}$ is the distributed force per unit mass, $\mathbf{T}^{\alpha}$ are stress vectors that contribute to the tractions transmitted across material curves, and the semi-colon is used to denote the surface covariant derivative using the metric of the coordinates induced by $\mathbf{r}\left(\theta^{\alpha}\right)$ [20]. The stress vectors are given by

$$
\mathbf{T}^{\alpha}=\mathbf{N}^{\alpha}+S^{\alpha} \mathbf{n},
$$

where $\mathbf{N}^{\alpha}$ are constitutively determined tangential vector fields, $S^{\alpha}$ is a contravariant vector field to be specified, and

$$
\mathbf{n}=\frac{1}{2} \varepsilon^{\alpha \beta} \mathbf{a}_{\alpha} \times \mathbf{a}_{\beta}
$$

is the local orientation of $\omega$. Here, $\mathbf{a}_{\alpha}=\mathbf{r}_{, \alpha}$ are the tangent vectors induced by the coordinates, commas denoting partial derivatives, $\varepsilon^{\alpha \beta}=a^{-1 / 2} e^{\alpha \beta}$ is the permutation tensor density, $e^{\alpha \beta}\left(=e_{\alpha \beta}\right)$ is the unit alternator $\left(e^{12}=+1\right)$, and $a=\operatorname{det}\left(a_{\alpha \beta}\right)$ where $a_{\alpha \beta}=\mathbf{a}_{\alpha} \cdot \mathbf{a}_{\beta}$ is the induced metric, non-negative definite in general and assumed here to be positive definite. It is well known that the surface divergence in (2.1) may be written $\mathbf{T}_{; \alpha}^{\alpha}=a^{-1 / 2}\left(a^{1 / 2} \mathbf{T}^{\alpha}\right)_{, \alpha}$, allowing one to avoid Christoffel symbols.

The constitutively determinate term in (2.2) is expressible in the form [20]

$$
\mathbf{N}^{\alpha}=N^{\beta \alpha} \mathbf{a}_{\beta},
$$

with

$$
N^{\beta \alpha}=\sigma^{\beta \alpha}+b_{\mu}^{\beta} M^{\mu \alpha},
$$

where

$$
\sigma^{\beta \alpha}=\rho\left(\frac{\partial \Psi}{\partial a_{\alpha \beta}}+\frac{\partial \Psi}{\partial a_{\beta \alpha}}\right), \quad M^{\beta \alpha}=\frac{\rho}{2}\left(\frac{\partial \Psi}{\partial b_{\alpha \beta}}+\frac{\partial \Psi}{\partial b_{\beta \alpha}}\right),
$$


and $b_{\alpha \beta}=\mathbf{n} \cdot \mathbf{r}_{, \alpha \beta}$ are the symmetric coefficients of the second fundamental form on $\omega$. The sign convention for $M^{\beta \alpha}$ differs from that commonly used in shell theory $[15,20]$, and the mixed components $b_{\beta}^{\alpha}$ in (2.5) are related to $b_{\alpha \beta}$ through $b_{\alpha \beta}=$ $a_{\alpha \lambda} b_{\beta}^{\lambda}$. Further, the coordinate-dependent function $\Psi\left(a_{\alpha \beta}, b_{\alpha \beta}\right)$ is the Galileaninvariant energy per unit mass of the film. I temporarily suppress the dependence of $\Psi$ on the particle $x$. The form of this function is such that its values are independent of the coordinate system as the energy is required to be an absolute scalar field [15, Section 13].

The local mass conservation law is

$$
\rho_{0}=J \rho, \quad \text { where } J=(a / A)^{1 / 2} ;
$$

$A$ and $\rho_{0}$ are the values of $a$ and $\rho$ respectively on a fixed reference surface $\Omega$.

Many writers study the response of fluid films subject to the two-dimensional incompressibility constraint $J=1$. This implies that deformations preserve surface area, and may be added to the list of constraints already imposed to obtain the local Kirchhoff-Love response functions from those of the Cosserat theory, as explained in [20]. The procedure used in that work yields equations identical to those obtained by using the formal Lagrange multiplier rule

$$
\Psi=\bar{\Psi}\left(a_{\alpha \beta}, b_{\alpha \beta}\right)-\gamma / \rho,
$$

in (2.6), where $\bar{\Psi}$ is a constitutive function and $\gamma\left(\theta^{\alpha}\right)$ is a constitutively indeterminate scalar field.

In the absence of distributed couples the normal components of (2.2) are given by

$$
S^{\alpha}=-M_{; \beta}^{\alpha \beta} .
$$

From the viewpoint of the constrained Cosserat theory, this follows from a director balance law which furnishes the values of the constitutively-indeterminate vector field $S^{\alpha}$ a posteriori [20].

In the present version of the theory, the moment-of-momentum balance is satisfied as an identity in accordance with conventional finite elasticity theory. NAGHDI $[15,22]$ used this balance law to determine the skew part of the coefficient matrix in (2.4).

The foregoing equations are well known in principle but rarely stated in forms that illuminate the underlying physics. In interpreting the various terms, it is helpful to relate them to the tractions and moments transmitted across material curves. To this end let $\theta^{\alpha}(s)$ be an arclength parametrization of such a curve on $\omega$ and let $\boldsymbol{\tau}$ be the unit tangent in the direction of increasing $s$. Then, $\boldsymbol{v}=\boldsymbol{\tau} \times \mathbf{n}$ is the rightward unit normal as the curve is traversed in the same direction. This has components $v_{\alpha}=\varepsilon_{\alpha \beta} \tau^{\beta}$ where $\tau^{\alpha}=\mathrm{d} \theta^{\alpha} / \mathrm{d} s$ are the components of $\tau$ and $\varepsilon_{\alpha \beta}=a^{1 / 2} e_{\alpha \beta}$ are the covariant components of the permutation tensor density. The traction transmitted by the material on the right to the material on the left is then given by [20]

$$
\mathbf{t}=\mathbf{T}^{\alpha} v_{\alpha}-\left(M^{\beta \alpha} v_{\alpha} \tau_{\beta} \mathbf{n}\right)^{\prime}
$$


where $\tau_{\alpha}=a_{\alpha \beta} \tau^{\beta}$ and the prime denotes the derivative with respect to $s$. This furnishes the force per unit arclength. One then uses (2.2) to interpret $S^{\alpha} v_{\alpha}$ as a transverse shear traction across the curve. The moment per unit length is [20]

$$
\mathbf{m}=\mathbf{r} \times \mathbf{t}-M \boldsymbol{\tau}, \text { where } M=M^{\beta \alpha} v_{\beta} v_{\alpha}
$$

is the bending couple.

Global forms of the equations for a simply-connected region $r \subset \omega$ are obtained by using Stokes' theorem in the form

$$
\int_{r} \mathbf{T}_{; \alpha}^{\alpha} \mathrm{d} a=\int_{\partial r} \mathbf{T}^{\alpha} v_{\alpha} \mathrm{d} s
$$

together with (2.1) and (2.10). The resulting force balance is [20]

$$
\int_{r} \rho \mathbf{f} \mathrm{d} a+\int_{\partial r} \mathbf{t} \mathrm{d} s+\sum \mathbf{g}_{i}=\mathbf{0},
$$

where

$$
\mathbf{g}=M^{\beta \alpha}\left[v_{\alpha} \tau_{\beta}\right] \mathbf{n}
$$

is the force acting at a vertex of $\partial r$ if the latter is piecewise smooth with a finite number of points where $\boldsymbol{\tau}$ and $\boldsymbol{v}$ are discontinuous; the sum ranges over all the vertices; and the notation [.] is used to denote the forward jump as a vertex is traversed in the sense of increasing $s$. Equation (2.13) is derived by regarding the second term on the right in (2.10) as a distributional derivative. Further, a straightforward but involved calculation [20] yields the global identity

$$
\int_{r} \rho \mathbf{r} \times \mathbf{f} \mathrm{d} a+\int_{\partial r} \mathbf{m} \mathrm{d} s+\sum \mathbf{r}_{i} \times \mathbf{g}_{i}=\mathbf{0},
$$

which may be regarded as the specialization to equilibrium of the moment-ofmomentum balance.

Most treatments $[9,17]$ of the Kirchhoff-Love equations are based on stationaryor minimum-energy considerations in the spirit of Kirchhoff's original work [16]. I record here an energy functional which is rendered stationary by films coexisting in equilibrium with bulk fluids in a gravity field. This energy is used in the discussion of necessary conditions for minimizing states in Section 7. It is sufficient for this purpose to confine attention to local compactly supported variations defined on a closed connected region $R$ consisting of the bulk fluids and a number of films. These films consist either of compact (closed) surfaces contained entirely within the interior of $R$, or of surfaces that intersect $\partial R$ along certain curves. In the latter case I avoid the complicated and irrelevant boundary terms associated with the film traction and moment by considering variations that vanish together with their gradients on $\partial R$.

The appropriate energy functional is

$$
E[\mathbf{y}, \mathbf{r}]=\sum_{i} \int_{v_{i}}[\Phi(\varrho(\mathbf{y}))+g \mathbf{k} \cdot \mathbf{y}] \varrho \mathrm{d} v+\sum_{j} \int_{\omega_{j}} \rho \Psi \mathrm{d} a,
$$


where $v_{i}$ and $\omega_{j}$ respectively are the volumes and surfaces occupied by the bulk fluids and films in $R, \Phi$ is the energy of a bulk fluid phase per unit mass, $\varrho(\mathbf{y})$ is the associated mass density, $g \mathbf{k}$ is the gravitational body force per unit mass (with $\mathbf{k}$ a fixed unit vector), and $\mathbf{y}$ is the spatial position of a bulk-fluid particle. The weights of the films are presumed negligible and the indices have been suppressed in the integrands to avoid cumbersome notation.

That equilibria render this energy stationary under the stated conditions may be verified by evaluating the Gâteaux differential of $E\left[\mathbf{y}_{\varepsilon}, \mathbf{r}_{\varepsilon}\right]$ with respect to a parameter $\varepsilon$ at the value $\varepsilon=0$ (say) associated with the equilibrium state. Here, $\mathbf{y}_{\varepsilon}$ and $\mathbf{r}_{\varepsilon}$ are the parametrized positions of particles of the bulk fluids and the films respectively. I refer to $[23,24]$ for details of the lengthy formal argument. In these works the elastic surface is regarded as being convected by the deformations of the medium with which it coexists, but the conclusions drawn remain valid under a weaker restriction appropriate to the system at hand. In particular, the differential of the bulk energy may be transformed to an expression involving surface integrals of the fluxes $P \mathbf{u} \cdot \hat{\mathbf{n}}$ over the boundaries of the bulk phases, where $P=\varrho^{2} \mathrm{~d} \Phi / \mathrm{d} \varrho$ is the pressure, $\hat{\mathbf{n}}$ is the exterior unit normal to the boundary of a typical phase, and $\mathbf{u}=\dot{\mathbf{y}}$ is the variation of $\mathbf{y}$, the superposed dot denoting the $\varepsilon$-derivative of $\mathbf{y}_{\varepsilon}$ at $\varepsilon=0$ [23]. A typical film $\omega$ constitutes part of the boundary between two such phases, each of which contributes a distinct flux, the associated normals having opposite senses. If $\mathbf{n}$ is the orientation field on $\omega$, the net flux is $[P \mathbf{u}] \cdot \mathbf{n}$, the bracket denoting jumps as $\omega$ is traversed in the direction of $\mathbf{n}$. For systems with monolayer or bilayer amphiphilic films, I assume that the bulk phases do not penetrate the films as the alternative would entail contact between water molecules and the water-repellent hydrophobic tail groups of the surfactant molecules. This implies that $\mathbf{u}_{ \pm} \cdot \mathbf{n}=\mathbf{v} \cdot \mathbf{n}$ where $\mathbf{v}=\dot{\mathbf{r}}$ is the variation of $\mathbf{r}$ on $\omega$ and $\mathbf{u}_{ \pm}$are the limits of $\mathbf{u}$ on either side. Thus $[\mathbf{u}] \cdot \mathbf{n}=0$ on $\omega$ and the net flux reduces to $[P] \mathbf{v} \cdot \mathbf{n}$. The stated equilibrium equations then follow from the fundamental lemma whether or not the film is a material surface with respect to the surrounding fluid as the tangential components of $\mathbf{v}$ need not be related to the tangential components of $\mathbf{u}_{ \pm}$.

To summarize, (2.16) is stationary at $\varepsilon=0$ if and only if (2.1) is satisfied on each film with $\rho \mathbf{f}=p \mathbf{n}$, where $p=-[P]$, together with

$$
\operatorname{grad} P=-\varrho g \mathbf{k}
$$

in each of the bulk phases [23], where $\operatorname{grad}(\cdot)$ is the gradient with respect to $\mathbf{y}$.

A standard formal argument yields the same results in the presence of two- or three-dimensional incompressibility constraints provided (2.8) and

$$
\Phi(\varrho)=-\hat{P}(\mathbf{X}) / \varrho
$$

are used in (2.16) as appropriate, where $\hat{P}$ is constitutively indeterminate,

$$
\varrho=\varrho_{0}(\mathbf{X}) / \operatorname{det}(\operatorname{Grad} \chi(X)),
$$

$\chi(X)$ is a one-to-one deformation function of a bulk phase with values $\mathbf{y}$ and fixed domain, $\varrho_{0}(\mathbf{X})$ is the fixed density at particle $\mathbf{X}, \operatorname{Grad}(\cdot)$ is the gradient with respect to $\mathbf{X}$, and $P=\hat{P}\left(\chi^{-1}(\mathbf{y})\right)[25,26]$. 
The difference between the functional (2.16) and any other that is rendered stationary by the local equilibrium equations involves null-Lagrangians that do not affect the conclusions of Section 7. These have been characterized by BALL [27] in a general context that incorporates the present model.

\section{Constitutive Theory}

In the foregoing I have mentioned only those concepts that are fundamental to the equilibrium of material surfaces under the stated hypotheses. In particular I have not discussed any material symmetry properties that fluid films may possess, since it appears to me that it is possible to pursue alternative developments according to whether or not films are assumed to possess such symmetry at the outset. Both are subsumed under elasticity. These concepts have been confused throughout the large literature on fluid films. The confusion appears to stem from the widespread tacit belief that material symmetry is equivalent to Galilean invariance. Further, from JENKINs' work [9] and from the analysis presented here, there emerges the fortuitous fact that films with fluid-like material symmetry have response functions that are formally the same as those of the alternative class.

Following NoLL [13], the idea that material bodies possess symmetry is normally phrased in terms of restrictions on the energy $\Psi$ associated with invariance of response under maps from some fixed local configuration to another. This generates an abstract symmetry group for the material manifold, which may then be used to obtain, via Noll's rule, the symmetry group relative to any fixed local configuration. Noll's theory has been adapted to material surfaces by MuRDoch \& CoHEN [14]. In this setting it becomes apparent that his concept is distinct from that of coordinate form invariance, according to which $\Psi$, regarded as a function of tensor components, is the same function of the components obtained by certain distinguished coordinate transformations. Form invariance is regarded as the primitive notion of symmetry in NAGHDI's work on shells [15] and in RIVLIN's formulation of finite elasticity theory [28].

I define fluidity in the framework of the Murdoch-Cohen theory in Section 4 and proceed to obtain a canonical energy function $\Psi$ for films that possess symmetry. In general the energy is defined on the body manifold, which in turn is parametrized by a global coordinate chart $\theta^{\alpha}$. In other words, a one-to-one correspondence may be established between each point $x$ of the (simply-connected) body manifold and fixed values of the pair of coordinates on a rectangular grid. Isometric immersions of this manifold into 3-space may then be described by the local surface parametrizations $\mathbf{r}\left(\theta^{\alpha}\right)$. To ensure that the energy is a scalar field on the body manifold, it is necessary that the form of the function $\Psi$ depend on the parametrization in such a way that its values do not.

One way to accommodate this requirement and the notion of fixed configuration embodied in the Noll-Murdoch-Cohen theory is to assume that

$$
\Psi\left(a_{\alpha \beta}, b_{\alpha \beta}\right)=\hat{\Psi}(\mathbf{C}, \boldsymbol{\kappa})
$$


where $\hat{\Psi}$ is a coordinate-independent function,

$$
\mathbf{C}=a_{\alpha \beta} \mathbf{A}^{\alpha} \otimes \mathbf{A}^{\beta}
$$

is the invertible symmetric surface strain tensor, and

$$
\boldsymbol{\kappa}=b_{\alpha \beta} \mathbf{A}^{\alpha} \otimes \mathbf{A}^{\beta}
$$

is the symmetric relative curvature tensor. This is the viewpoint adopted in most works on elastic surfaces. In these definitions $\mathbf{A}^{\alpha}$ are dual vectors to the induced tangent vectors $\mathbf{A}_{\alpha}=\mathbf{x}_{, \alpha}$ at the particle $x$, where $\mathbf{x}\left(\theta^{\alpha}\right)$ is the local parametrization of a reference surface $\Omega$. Given the parametrization, these tensors, together with $\hat{\Psi}$, are functions of the matrices $a_{\alpha \beta}$ and $b_{\alpha \beta}$ as suggested by the notation of (3.1).

MuRDOCH \& COHEN introduced a primitive notion of material surface that includes (3.1) as a special case. In their work the local constitutive response is defined by Galilean-invariant functions of

$$
\mathbf{F}=\mathbf{a}_{\alpha} \otimes \mathbf{A}^{\alpha}
$$

and its gradient. This maps the tangent space of $\Omega$ to that of $\omega$ at $x$. For hyperelastic surfaces, their constitutive equations follow from (3.1) if dependence on strain gradient is suppressed.

The notion that elastic bodies possess symmetry of one kind or another is so widespread that it seems to be regarded as fundamental. In this work I regard it simply as a constitutive assumption. What is fundamental is the requirement that the energy $\Psi$ be a scalar field on the body manifold, and there are other ways to ensure this. For example, one notion entirely compatible with the general theory of material surfaces is that $\Psi$ is sensitive only to the local configuration of the film in a neighborhood of $x$ rather than to the kinematical variables that describe its deformations.

A similar premise underlies the conventional theory of single-phase liquidcrystal equilibria [29], in which a director field defining the crystalline orientation is described by the Euler equations associated with an energy that depends on the local values of the director and its spatial gradient. There is no dependence on the kinematics of director deformation or on the deformation of the fluid medium. Indeed the director configurations are deemed to be independent of the deformations of the fluid in the commonly accepted formulations [29,30]. The canonical form of the energy function is then deduced from Galilean invariance and any additional restrictions deemed to be relevant in particular circumstances, but these are unrelated to symmetry in Noll's sense.

For films, one may base such a theory on the premise that the energy per unit mass is a function of the surface density $\rho$ and the symmetric surface curvature tensor

$$
\mathbf{b}=b_{\alpha \beta} \mathbf{a}^{\alpha} \otimes \mathbf{a}^{\beta}=-\mathbf{n}_{, \alpha} \otimes \mathbf{a}^{\alpha},
$$

where the $\mathbf{a}^{\alpha}$ are the duals to the $\mathbf{a}_{\alpha}$ on the tangent planes of $\omega$. The energy then responds only to the local shape and density of $\omega$. Galilean invariance requires that it be unaffected by the replacement of $\mathbf{b}$ with $\mathbf{Q b} \mathbf{Q}^{t}$ for all $\mathbf{b}$ in its domain, 
where $\mathbf{Q}$ is orthogonal and the superscript $t$ is used to denote transposition. Here I take this domain to consist of all ordered pairs of positive scalars and symmetric surface tensors defined on the tangent space of $\omega$ at the particle in question. For the invariance condition to make sense it is then necessary to restrict $\mathbf{Q}$ such that $\mathbf{a}^{\alpha}$ and $\mathbf{Q} \mathbf{a}^{\alpha}$ span the same vector space. This restriction entails no loss of generality since the rotational invariance of the energy implies that, at a given particle, the tangent space obtained by rotating $\omega$ may be identified with the tangent space to $\omega$ itself insofar as the constitutive response is concerned [14]. The $\mathbf{Q}$ appearing in the invariance condition should then be interpreted as the projection onto the tangent space of an arbitrary three-dimensional rotation. As such it is a two-dimensional rotation if the three-dimensional rotation preserves the local orientation of $\omega$ and a two-dimensional orthogonal transformation in the general case. This form of the invariance condition is equivalent to that adopted by MoECKEL [31, Section 6(c)] in his thermodynamical theory of interfaces.

Galilean invariance is thus tantamount to the invariance of the energy, regarded as a function of a symmetric two-dimensional tensor, under arbitrary twodimensional orthogonal transformations. For this it is necessary and sufficient that the energy be expressible in the form [32]

$$
\Psi\left(a_{\alpha \beta}, b_{\alpha \beta}\right)=F(\rho, H, K),
$$

where

$$
H=\frac{1}{2} \operatorname{tr} \mathbf{b}, \quad K=\operatorname{det} \mathbf{b}
$$

are the mean and Gaussian curvatures of $\omega$ respectively. This in turn may be expressed as a function of the matrices $a_{\alpha \beta}$ and $b_{\alpha \beta}$, as indicated, by using the local mass conservation law (2.7) and the formulae

$$
H=\frac{1}{2} a^{\alpha \beta} b_{\alpha \beta} \text { and } K=\frac{1}{2} \varepsilon^{\alpha \beta} \varepsilon^{\lambda \mu} b_{\alpha \lambda} b_{\beta \mu},
$$

where $\left(a^{\alpha \beta}\right)=\left(a_{\alpha \beta}\right)^{-1}$ is the dual metric, $\varepsilon^{\alpha \beta}=a^{-1 / 2} e^{\alpha \beta}$ and $a=\operatorname{det}\left(a_{\alpha \beta}\right)$. Therefore this alternative constitutive formulation, essentially equivalent to that introduced by BLINOWsKI [33], also furnishes a model for material surfaces. It is similar in some respects to a theory proposed by FAETTI \& VIRGA [34] for liquid crystals with surface energy, provided that the fluid orientation is aligned locally with the surface normal. Related theories had been proposed earlier by JENKINS \& BARRAT [35] and ERICKSEN [36].

\section{Films with material symmetry}

Material symmetry theory for surfaces is not settled. This appears to be due to the difference between form invariance with respect to distinguished coordinate transformations and Noll's invariance of response under distinguished compositions of maps. This distinction is easily overlooked in the conventional theory of simple materials as the two concepts then lead to mathematically identical problems. For plate and shell theories, a number of alternative proposals have been advanced, 
some incorporating elements of NoLL's approach [14, 37, 38]. Among them, I find that of Murdoch \& COHEN [14] to be the most satisfactory extension of Noll's concept. This is based on the notion that local configurations of the body are to be regarded as the restrictions to surfaces of diffeomorphisms of 3-space. Symmetries are associated with local maps among fixed surfaces that leave the energy invariant in a given diffeomorphism. The implications of this idea for elastic surface-substrate interactions have been examined in [24]. I present a brief summary of the Murdoch-Cohen theory here, with adaptations tailored to the narrower class of material surfaces considered.

Preliminary to this, I examine certain local properties of maps between two fixed surfaces $\Omega$ and $\Omega^{*}$, with local parametrizations $\mathbf{x}\left(\theta^{\alpha}\right)$ and $\mathbf{x}^{*}\left(\theta^{\alpha}\right)$ respectively, occupied by the same material body. Thus, let $\phi(\mathbf{X})$ be a $C^{2}$ orientation-preserving diffeomorphism of 3-space to itself defined on an open neighborhood of a material point $x$ with coordinates $\theta^{\alpha}$. Let $N^{*} \subset \Omega^{*}$ be the intersection of this neighborhood with $\Omega^{*}$, and suppose $\mathbf{x}=\phi\left(\mathbf{x}^{*}\right)$ for $\mathbf{x}^{*} \in N^{*}$. Then, $N=\phi\left(N^{*}\right) \subset \Omega$ is the intersection of the same set of material points with $\Omega$.

If $\mathbf{A}_{\alpha}^{*}$ and $\mathbf{A}_{\alpha}$ are the tangent vectors induced by $\theta^{\alpha}$ on $\Omega^{*}$ and $\Omega$ at $x$, then

$$
\mathbf{A}_{\alpha}=(\nabla \phi) \mathbf{A}_{\alpha}^{*} \quad \text { and } \quad \mathbf{A}_{\alpha, \beta}=(\nabla \nabla \phi)\left[\mathbf{A}_{\alpha}^{*} \otimes \mathbf{A}_{\beta}^{*}\right]+(\nabla \phi) \mathbf{A}_{\alpha, \beta}^{*},
$$

where $\nabla \phi$ and $\nabla \nabla \phi$ are the first and second gradients of $\phi(\mathbf{X})$ evaluated at $x \in N^{*}$. The operation in the second expression is defined, using Cartesian notation, by

$$
(\nabla \nabla \phi)[\mathbf{u} \otimes \mathbf{v}]=\left(\partial^{2} \phi_{i} / \partial X_{A} \partial X_{B}\right) u_{A} v_{B} \mathbf{e}_{i},
$$

with $\left\{\mathbf{e}_{i}\right\}$ an orthonormal basis for 3-space.

In the remainder of this section I assume the tangent spaces to the various surfaces occupied by the body to coincide at the particle $x$. Galilean invariance implies that this entails no loss of generality in the characterization of constitutive response [14]. With this adjustment (4.1) 1 is then equivalent to

$$
\mathbf{A}_{\alpha}=\mathbf{H A}_{\alpha}^{*}=H_{\cdot \alpha}^{\lambda} \mathbf{A}_{\lambda}^{*},
$$

where

$$
\mathbf{H}=\mathbf{A}_{\alpha} \otimes \mathbf{A}^{* \alpha}=H_{\cdot \beta}^{\alpha} \mathbf{A}_{\alpha}^{*} \otimes \mathbf{A}^{* \beta}, \quad H_{\cdot \beta}^{\alpha}=\mathbf{A}^{* \alpha} \cdot(\nabla \phi) \mathbf{A}_{\beta}^{*},
$$

and the $\mathbf{A}^{* \alpha}$ are dual to $\mathbf{A}_{\alpha}^{*}$. The properties of $\phi$ ensure that $\mathbf{H}$ is an invertible linear transformation from the tangent space to itself. Accordingly, there is a tensor $\mathbf{R}$ of the same type such that $\mathbf{R}^{t}=\mathbf{H}^{-1}$, and it is straightforward to show that

$$
\mathbf{A}^{\alpha}=\mathbf{R A}^{* \alpha} .
$$

Let $\mathbf{N}$ be the orientation of $\Omega$ at $x$. Then,

$$
\mu_{\alpha \beta} \mathbf{N}=\mathbf{A}_{\alpha} \times \mathbf{A}_{\beta},
$$


where $\mu_{\alpha \beta}=A^{1 / 2} e_{\alpha \beta}$ is the associated permutation tensor density. I combine this with a similar formula for the orientation $\mathbf{N}^{*}$ of $\Omega^{*}$ and use (4.3) with $\mu_{\lambda \gamma}^{*} H_{\cdot \alpha}^{\lambda} H_{\cdot \beta}^{\gamma}=$ $(\operatorname{det} \mathbf{H}) \mu_{\alpha \beta}^{*}$ to derive

$$
\mu_{\alpha \beta} \mathbf{N}=(\operatorname{det} \mathbf{H}) \mu_{\alpha \beta}^{*} \mathbf{N}^{*} .
$$

This result and $A / A^{*}=(\operatorname{det} \mathbf{H})^{2}$, which follows from (4.3), yield

$$
\operatorname{det} \mathbf{H}= \pm\left(A / A^{*}\right)^{1 / 2}
$$

according as $\mathbf{N}= \pm \mathbf{N}^{*}$. In particular, $\operatorname{det} \mathbf{R}(=1 / \operatorname{det} \mathbf{H})$ is positive if and only if $\Omega$ and $\Omega^{*}$ have the same orientation; otherwise it is negative.

Let $\mathbf{B}$ be the curvature tensor of $\Omega$ at $x$. Then $\mathbf{B}=B_{\alpha \beta} \mathbf{A}^{\alpha} \otimes \mathbf{A}^{\beta}$, where $B_{\alpha \beta}=$ $\mathbf{N} \cdot \mathbf{A}_{\alpha, \beta}$. Let $\mathbf{B}^{*}$, defined similarly, be the curvature tensor of $\Omega^{*}$ at the same particle. The relationship between the two curvatures may be inferred from $(4.1)_{1,2}$, (4.5) and the fact that $\nabla \phi$ maps the tangent space to itself:

$$
\mathbf{R}^{-1} \mathbf{B} \mathbf{R}^{-t}=\left(\mathbf{N} \cdot \nabla \nabla \phi\left[\mathbf{A}_{\alpha} \otimes \mathbf{A}_{\beta}\right]\right) \mathbf{A}^{\alpha} \otimes \mathbf{A}^{\beta}+\left(\mathbf{N} \cdot(\nabla \phi) \mathbf{N}^{*}\right) \mathbf{B}^{*},
$$

wherein the first term on the right has the same value regardless of which set of tangent bases is used.

Consider a configuration $\omega$ of the film parametrized locally by $\mathbf{r}\left(\theta^{\alpha}\right)$, and let $\mathbf{C}$, $\boldsymbol{\kappa}$ and $\mathbf{C}^{*}, \boldsymbol{\kappa}^{*}$ be the strains and curvatures of $\omega$ relative to $\Omega$ and $\Omega^{*}$ respectively. Since the first and second fundamental forms on $\omega$ are determined by its parametric representation, it follows from (3.2), (3.3) and (4.5) that

$$
\mathbf{C}=\mathbf{R C}^{*} \mathbf{R}^{t} \text { and } \boldsymbol{\kappa}=R \boldsymbol{\kappa}^{*} \mathbf{R}^{t} .
$$

The energy per unit mass is presumed to be a property of the body in a given state. As such, its values at $x$ are not dependent on the reference surface used to compute them. In the notation of (3.1),

$$
\hat{\Psi}^{*}\left(\mathbf{C}^{*}, \boldsymbol{\kappa}^{*}\right)=\hat{\Psi}(\mathbf{C}, \boldsymbol{\kappa})=\hat{\Psi}\left(\mathbf{R} \mathbf{C}^{*} \mathbf{R}^{t}, \mathbf{R} \kappa^{*} \mathbf{R}^{t}\right),
$$

where $\hat{\Psi}$ and $\hat{\Psi}^{*}$ are constitutive functions defined on $\Omega$ and $\Omega^{*}$.

Consider another diffeomorphism $\xi(\mathbf{X})$ of the same kind as $\boldsymbol{\phi}$ but with the property that $\mathbf{r}=\xi(\mathbf{x})$ for $\mathbf{x} \in N$. This induces at $x$ a strain

$$
\mathbf{C}=\left((\nabla \boldsymbol{\xi}) A_{\alpha} \cdot(\nabla \boldsymbol{\xi}) A_{\beta}\right) \mathbf{A}^{\alpha} \otimes \mathbf{A}^{\beta}
$$

relative to $\Omega$ and a relative curvature obtained with the aid of the formula

$$
\boldsymbol{\kappa}=F^{t} \mathbf{b F},
$$

which follows from (3.3)-(3.5). With the tangent spaces aligned at $x, \mathbf{F}$ plays the same role in the local map from $\Omega$ to $\omega$ as that played by $\mathbf{H}$ in the map from $\Omega^{*}$ to $\Omega$. Accordingly, (4.8) and (4.12) give

$$
\boldsymbol{\kappa}=\left(\mathbf{n} \cdot \nabla \nabla \boldsymbol{\xi}\left[\mathbf{A}_{\alpha} \otimes \mathbf{A}_{\beta}\right]\right) \mathbf{A}^{\alpha} \otimes \mathbf{A}^{\beta}+(\mathbf{n} \cdot(\nabla \boldsymbol{\xi}) N) B,
$$


where $\mathbf{n}$ is the orientation of $\omega$ at $x$. For definiteness, and (by Galilean invariance) without loss of generality [14], I choose the orientations of the reference and distorted surfaces to coincide at $x$. Thus, $\mathbf{n}=\mathbf{N}$ in (4.13).

To characterize the relationship between $N$ and $N^{*}$ due to symmetry, it is necessary to determine the strain $\overline{\mathbf{C}}$ and curvature $\overline{\boldsymbol{\kappa}}$ relative to $\Omega^{*}$ induced at $x$ by $\mathbf{r}^{*}=\xi\left(\mathbf{x}^{*}\right)$ for $\mathbf{x}^{*} \in N^{*}$. These are given by the obvious modifications to (4.11) and (4.13) in which $\mathbf{n}^{*}=\mathbf{N}^{*}$. The values of $\nabla \boldsymbol{\xi}$ and $\nabla \nabla \boldsymbol{\xi}$ are the same in both sets of formulas, and it follows easily that the strains are also equal, but $\overline{\boldsymbol{\kappa}}$ and $\boldsymbol{\kappa}$ differ in a manner that depends on the relative orientations of $N^{*}$ and $N$ :

$$
\overline{\mathbf{C}}=\mathbf{C}, \quad \overline{\boldsymbol{\kappa}}=\left\{\begin{array}{cc}
\kappa+(\mathbf{N} \cdot(\nabla \boldsymbol{\xi}) \mathbf{N})\left(\mathbf{B}^{*}-\mathbf{B}\right) ; & \operatorname{det} \mathbf{R}>0 \\
-\boldsymbol{\kappa}+(\mathbf{N} \cdot(\nabla \boldsymbol{\xi}) \mathbf{N})\left(\mathbf{B}^{*}+\mathbf{B}\right) ; & \operatorname{det} \mathbf{R}<0 .
\end{array}\right\},
$$

wherein $\mathbf{B}$ and $\mathbf{B}^{*}$ are connected by the map $\phi(X)$ through (4.8).

Following Noll [13], Murdoch \& CoHen [14] regard $N$ and $N^{*}$ as being related by symmetry if they respond identically to the same $\boldsymbol{\xi}(\mathbf{X})$. Then, $\hat{\Psi}(\mathbf{C}, \boldsymbol{\kappa})=$ $\boldsymbol{\Psi}^{*}(\overline{\mathbf{C}}, \overline{\boldsymbol{\kappa}})$, which, when combined with (4.10), yields

$$
\hat{\Psi}(\mathbf{C}, \kappa)=\hat{\Psi}\left(\mathbf{R} \overline{\mathbf{C}} \mathbf{R}^{t}, \mathbf{R} \bar{\kappa} \mathbf{R}^{t}\right) .
$$

This is identically satisfied for all $\mathbf{C}$ and $\boldsymbol{\kappa}$ in the domain of $\hat{\Psi}$ if $\phi$ is the identity map with $\nabla \nabla \boldsymbol{\phi}=\mathbf{0}$ and $\nabla \boldsymbol{\phi}=\mathbf{I}$, the unit tensor for 3-space, and $\mathbf{R}$ the projection of $\mathbf{I}$ onto the tangent space at $x\left(\operatorname{det} \mathbf{R}=1, \mathbf{N}^{*}=\mathbf{N}, \mathbf{B}^{*}=\mathbf{B}\right)$. However, it is not at all certain that there exist other maps $\phi$ with the required properties for arbitrary surfaces. General surfaces may therefore be expected to have only trivial symmetry. Particular surfaces with non-trivial symmetries specific to solid films have been studied in [24].

Murdoch \& CoHen have shown that the pairs $\left(\mathbf{R}, \mathbf{B}^{*} \pm \mathbf{B}\right)$ satisfying (4.15), with the sign chosen in accordance with (4.14), are elements of a group. Thus, arguments used in conventional elasticity may be used here to restrict the entries $\mathbf{R}$ to unimodular (surface) tensors $(\operatorname{det} \mathbf{R}= \pm 1$ ).

The structure of amphiphilic bilayers and the high degree of in-plane mobility observed in equilibrium states suggest a definition of fluidity analogous to that of NoLL [13] for conventional bulk fluids. Thus, suppose the embedding geometry of $N^{*} \subset \Omega^{*}$ is that of a plane, so that $\mathbf{B}^{*}=\mathbf{0}$ at $x$. For such $N^{*}$, I define fluidity by the requirement that (4.15) be satisfied for all affine $\phi(\mathbf{X})(\nabla \nabla \phi \equiv \mathbf{0})$ with the properties that $\nabla \phi$ is proper unimodular $(\operatorname{det} \nabla \phi=+1)$ and maps the subspaces $\mathcal{T}$ and $\operatorname{span}\{\mathbf{N}\}$ to themselves, where $\mathcal{T}$ is the common tangent space at $x$. The induced surface tensor $\mathbf{R}$ fulfills the requirement $|\operatorname{det} \mathbf{R}|=1$, and (4.8) implies that $N$ is related to $N^{*}$ by symmetry only if $\mathbf{B}=\mathbf{0}$. Accordingly, $(4.14)_{2}$ simplifies to $\overline{\boldsymbol{\kappa}}= \pm \boldsymbol{\kappa}$ and (4.15) becomes

$$
\hat{\Psi}(\mathbf{C}, \boldsymbol{\kappa})=\hat{\Psi}\left(\mathbf{R C R}^{t}, \pm \mathbf{R} \kappa R^{t}\right) ; \quad \operatorname{det} \mathbf{R}= \pm 1 .
$$

This definition of fluidity is meant to reflect the small-scale three-dimensional structure of bilayers in configurations in which the interfaces between the bilayer and the bulk fluid are parallel planes, as depicted, for example, in [5, Figure 1.1] 
and in [12, Figure 4.2]. Its implications for response in arbitrary configurations are of primary interest here.

To obtain the canonical form of the energy function, I note that surface rotations $\left(\mathbf{R}^{-1}=\mathbf{R}^{t}\right.$, $\left.\operatorname{det} \mathbf{R}=+1\right)$ are admitted by the definition. The appropriate specialization of (4.16) is satisfied for all such $\mathbf{R}$ if and only if $\hat{\Psi}$ is expressible as a function of the elements of the hemitropic function basis [32]:

$$
I=\{\operatorname{tr} \mathbf{C}, \operatorname{det} \mathbf{C}, \operatorname{tr} \boldsymbol{\kappa}, \operatorname{det} \boldsymbol{\kappa}, \operatorname{tr}(\mathbf{C} \boldsymbol{\kappa}), \operatorname{tr}(\mathbf{C} \kappa \boldsymbol{\mu})\},
$$

where $\boldsymbol{\mu}=\mu_{\alpha \beta} \mathbf{A}^{\alpha} \otimes \mathbf{A}^{\beta}$. Invariance under arbitrary proper-unimodular transformations implies that the energy is expressible as a function of all independent invariants formed from the list (4.17) that are also proper-unimodular invariants. That this class of functions is the most general one to fulfill the stated requirement follows from the fact that invariance under the larger set of transformations implies invariance under the smaller set. Thus, while any such function is necessarily expressible in terms of the elements of the set $I$, it cannot be an arbitrary function of these elements.

The obvious candidates for inclusion are $\operatorname{det} \mathbf{C}$ and $\operatorname{det} \boldsymbol{\kappa}$. Another invariant having the required property is

$$
\sigma=(\operatorname{tr} \mathbf{C})(\operatorname{tr} \boldsymbol{\kappa})-\operatorname{tr}(\mathbf{C} \boldsymbol{\kappa}) .
$$

To prove this I use the Cayley-Hamilton theorem for arbitrary symmetric surface tensors A:

$$
\tilde{\mathbf{A}}=(\operatorname{tr} \mathbf{A}) \mathbf{1}-\mathbf{A},
$$

where $\tilde{\mathbf{A}}$ is the adjugate of $\mathbf{A}$ and $\mathbf{1}$ is the unit tensor for the fixed tangent space at $x$. Thus,

$$
\sigma=\operatorname{tr}(\tilde{\mathbf{C}} \boldsymbol{\kappa})=\operatorname{tr}(\mathbf{C} \tilde{\boldsymbol{\kappa}}) .
$$

Since $\mathbf{C}$ is presumed invertible, the first alternative gives $\sigma=(\operatorname{det} \mathbf{C}) \operatorname{tr}\left(\mathbf{C}^{-1} \boldsymbol{\kappa}\right)$, in which the second factor is invariant under the replacements $\mathbf{C} \rightarrow \mathbf{R} \mathbf{C} \mathbf{R}^{t}$ and $\boldsymbol{\kappa} \rightarrow$ $\mathbf{R} \boldsymbol{\kappa} \mathbf{R}^{t}$ for all invertible surface tensors $\mathbf{R}$. The result then follows by the unimodular invariance of det $\mathbf{C}$. I have not succeeded in generating additional independent proper-unimodular invariants from the set $I$ and thus conjecture that the three discussed comprise the maximal set.

It is easily demonstrated that $\operatorname{det} \mathbf{C}=J^{2}$. Granted the truth of the conjecture, it is thus necessary that

$$
\begin{aligned}
\hat{\Psi}(\mathbf{C}, \boldsymbol{\kappa}) & =G(J(\mathbf{C}), \sigma(\mathbf{C}, \boldsymbol{\kappa}), \kappa(\boldsymbol{\kappa}) ; x) \\
J(\mathbf{C}) & \doteq(\operatorname{det} \mathbf{C})^{1 / 2}, \quad \sigma(\mathbf{C}, \boldsymbol{\kappa}) \doteq \operatorname{tr}(\tilde{\mathbf{C}} \boldsymbol{\kappa}), \quad \kappa(\boldsymbol{\kappa}) \doteq \operatorname{det} \boldsymbol{\kappa},
\end{aligned}
$$

where $G$ is scalar valued and parametric dependence on the particle is indicated explicitly. Conversely, if (4.21) holds, then, since $\kappa$ and $\sigma$ are even and odd functions of $\boldsymbol{\kappa}$ respectively, (4.16) is satisfied for all unimodular $\mathbf{R}(\operatorname{det} \mathbf{R}= \pm 1$ ) provided that $G$ is an even function of $\sigma$. Each of these generates a proper-unimodular $\nabla \boldsymbol{\phi}$ 
through $\nabla \boldsymbol{\phi}=\mathbf{R}^{-t} \pm \mathbf{N} \otimes \mathbf{N}$, with the sign chosen as appropriate. This in turn is the general form of $\nabla \boldsymbol{\phi}$ compatible with the definition of fluidity and so the bilayer is fluid if and only if (4.21) holds with $\sigma$ replaced by $|\sigma|$.

A monolayer film may be viewed as half a bilayer. For these an appropriate definition of fluidity is obtained from that for bilayers by restricting $\phi$ so as to preserve the local orientation of the surface. In this case the second branch of (4.16) is not applicable and the necessary and sufficient condition for fluidity is again given by (4.21), but without the requirement that $G$ be an even function of $\sigma$.

To determine the response functions relative to arbitrary local reference configurations, I re-write (4.21) in the form

$$
\begin{aligned}
\hat{\Psi}_{\lambda}\left(\mathbf{C}_{\lambda}, \boldsymbol{\kappa}_{\lambda}\right) & =G_{\lambda}\left(J_{\lambda}, \sigma_{\lambda}, \kappa_{\lambda} ; x\right) ; \quad J_{\lambda}=J\left(\mathbf{C}_{\lambda}\right), \\
\sigma_{\lambda} & =\sigma\left(\mathbf{C}_{\lambda}, \boldsymbol{\kappa}_{\lambda}\right), \quad \kappa_{\lambda}=\kappa\left(\boldsymbol{\kappa}_{\lambda}\right),
\end{aligned}
$$

where the subscript $\lambda$ is used to identify the reference configuration. With $\omega$ fixed, the transformation from $\lambda$ to an another local reference configuration, $\mu$ say, yields the composition formula $J_{\lambda}=J_{\mu} D(x)$, where $J_{\mu}=J\left(\mathbf{C}_{\mu}\right)$ and $D(x)$ is the positive square root of the determinant of the strain of $\mu$ relative to $\lambda$ at $x$. Next, I observe that $\kappa$ is related to $\boldsymbol{\kappa}$ in the same way that the Gaussian curvature $K$ is related to $\mathbf{b}$ (cf. (3.7) and (3.8)). Thus,

$$
\kappa=\frac{1}{2} \mu^{\alpha \beta} \mu^{\lambda \mu} b_{\alpha \lambda} b_{\beta \mu}=J^{2} K,
$$

and so $\kappa_{\lambda}=\kappa_{\mu} D^{2}$, where $\kappa_{\mu}=\kappa\left(\kappa_{\mu}\right)$. The function $\sigma$ may likewise be expressed in terms of the mean curvature $H$ by using (3.3) and $\mathbf{C}^{-1}=a^{\alpha \beta} \mathbf{A}_{\alpha} \otimes \mathbf{A}_{\beta}$ in (4.20). Comparison of the result with $(3.8)_{1}$ gives

$$
\sigma=2 J^{2} H
$$

Hence, $\sigma_{\lambda}=\sigma_{\mu} D^{2}$, where $\sigma_{\mu}=\sigma\left(\mathbf{C}_{\mu}, \boldsymbol{\kappa}_{\mu}\right)$.

If $\hat{\Psi}_{\mu}$ is the response function with $\mu$ as reference, then

$$
\hat{\Psi}_{\mu}\left(\mathbf{C}_{\mu}, \boldsymbol{\kappa}_{\mu}\right)=\hat{\Psi}_{\lambda}\left(\mathbf{C}_{\lambda}, \boldsymbol{\kappa}_{\lambda}\right),
$$

and (4.22) furnishes

$$
\hat{\Psi}_{\mu}=G_{\mu}\left(J_{\mu}, \sigma_{\mu}, \kappa_{\mu} ; x\right)
$$

where

$$
G_{\mu}\left(J_{\mu}, \sigma_{\mu}, \kappa_{\mu} ; x\right)=G_{\lambda}\left(J_{\mu} D(x), \sigma_{\mu} D^{2}(x), \kappa_{\mu} D^{2}(x) ; x\right) .
$$

This holds without restrictions on the embedding geometry of $\mu$ or its tangent space. Equation (4.21) therefore implies that the response relative to any local reference configuration is sensitive to the strain and relative curvature through the associated values of $J, \sigma$ and $\kappa$. 


\section{Alternative formulations}

JENKINS [9] claimed that (3.6) is the necessary and sufficient condition for a material film with symmetry to qualify as fluid by a standard which, though conceptually different from that used here, nevertheless imposes the same mathematical restrictions on the energy. To my knowledge he is the only writer on the subject of films to have investigated the consequences of fluidity as a restriction associated with material symmetry rather than with Galilean invariance. His analysis combines the elements of coordinate form invariance as advocated by NAGHDI [15] with the well-known procedure used in Noll's theory of simple elastic materials to derive the canonical constitutive equation for the energy in compressible bulk fluids. The latter procedure was also used by COHEN \& WANG [39] to obtain the general strain-energy function for fluid membranes with symmetry. Detailed criticism of this procedure in the context of compressible bulk-fluid response has been given by RIVLIN \& SMITH [40]. However, in that theory, as in the present theory of fluid films, the logical errors in the analysis do not invalidate the conclusions.

Jenkins' model is based on a constitutive framework whose Galilean-invariant specialization is equivalent to (3.1). To model fluidity, he assumes the invariance of the energy per unit mass under coordinate transformations with unimodular matrices of partial derivatives; this he represents by (4.3) with $|\operatorname{det} \mathbf{H}|=1$. For Galileaninvariant energies, his concept of symmetry, which involves no restrictions on the local embedding geometry of the reference surface, is then equivalent to (4.16), but with the plus sign used in the argument of the right-hand side for general unimodu$\operatorname{lar} \mathbf{R}$. This minor discrepancy is of no consequence here since Jenkins' argument is based on proper-unimodular transformations; (4.21) was likewise derived by using the corresponding branch of (4.16). Accordingly, the problem of deriving reduced forms of the energy that are necessary and sufficient for fluidity is essentially the same in both theories, granted the Galilean invariance of the energy.

To pursue the question, I note that the tensor $\mathbf{F}$ defined by (3.4) possesses a determinant if the tangent spaces of the reference and distorted surfaces coincide at $x$, this then being equal to $\pm J$. Then, $\mathbf{H}=J^{-1 / 2} \mathbf{F}$ is a proper-unimodular surface tensor if the surfaces also have the same orientation. It furnishes the basis for Jenkins' discussion of necessary and sufficient conditions for fluidity, in which the response function is not presumed to be Galilean invariant a priori. In the present context this choice corresponds to $\mathbf{R}=J^{1 / 2} \mathbf{F}^{-t}$, which I use with (4.12) and the relevant branch of (4.16) to obtain the apparent necessary condition

$$
\hat{\Psi}(\mathbf{C}, \boldsymbol{\kappa})=\hat{\Psi}(J \mathbf{1}, J \mathbf{b}) \doteq \Sigma(J, \mathbf{b}), \quad \text { say, }
$$

where $\mathbf{1}$ is the fixed unit tensor for the considered tangent space and the relation $\mathbf{C}=\mathbf{F}^{t} \mathbf{F}$ has been used. The fluid film thus possesses flexural resistance by virtue of the dependence of its energy on the curvature b. That (4.21) is consistent with this conclusion follows from (3.7), (4.23) and (4.24). No further conclusions can be drawn from (4.16) and (5.1) since $\mathbf{b}$ is independent of the reference configuration. Thus, to conclude that (5.1) is equivalent to (3.6) it would appear to be necessary to subject the function $\Sigma$ to the further requirement of Galilean invariance. Of course, this reasoning cannot be correct since $\Sigma$ furnishes the values of a Galilean-invariant 
function by definition, and, as such, is automatically invariant. The logical error, as noted by RIVLIN \& SMITH in a similar context, may be traced to the failure to recognize that, in the discussion of necessary conditions for (4.16), $\mathbf{R}$ must be regarded as a fixed tensor if this equation is to be meaningful. Here, the error leads to the paradoxical conclusion that a Galilean-invariant energy is necessarily a function of a tensor which is sensitive to superposed rigid rotations.

To correct the argument, I replace $\mathbf{F}$ by $\mathbf{F}^{\prime}=\mathbf{a}_{\alpha}^{\prime} \otimes \mathbf{A}^{\alpha}$, the gradient of the map from $\Omega$ to a fixed surface $\omega^{\prime}$ that coincides with $\omega$ in a particular (but arbitrary) deformation. $\mathbf{F}^{\prime}$ is equal to $\mathbf{F}$ for this deformation, but, unlike $\mathbf{F}$, is unaffected by further deformation of the film or by superposed rigid motion. The use of the fixed proper-unimodular tensor $\mathbf{R}^{\prime}=\sqrt{J^{\prime}}\left(\mathbf{F}^{\prime}\right)^{-t}$ in (4.16), where $J^{\prime}=\operatorname{det} \mathbf{F}^{\prime}$, then yields the necessary condition

$$
\hat{\Psi}(\mathbf{C}, \boldsymbol{\kappa})=\hat{\Psi}\left(J^{\prime} \mathbf{1}^{\prime}, J^{\prime} \mathbf{b}^{\prime}\right)
$$

in place of (5.1), where $\mathbf{1}^{\prime}=a_{\alpha \beta} \mathbf{a}^{\prime \alpha} \otimes \mathbf{a}^{\prime \beta}, \mathbf{b}^{\prime}=b_{\alpha \beta} \mathbf{a}^{\prime \alpha} \otimes \mathbf{a}^{\prime \beta}$ and the $\mathbf{a}^{\prime \alpha}$ are dual to $\mathbf{a}_{\alpha}^{\prime}$. These are just the strain and curvature of $\omega$ relative to $\omega^{\prime}$, the values of $\mathbf{C}$ and $\boldsymbol{\kappa}$ with $\omega^{\prime}$ as reference. As such they are equal to $\mathbf{1}$ and $\mathbf{b}$ in the considered deformation, but are unaffected by superposed rigid motion. That (5.2) fails to furnish a sufficient condition for the first branch of (4.16) to be satisfied may be demonstrated by choosing $\mathbf{R}=\overline{\mathbf{R}} \mathbf{R}^{\prime}$ with $\overline{\mathbf{R}}$ proper unimodular. Then, from (5.2), the relevant branch of (4.16) is seen to be equivalent to

$$
\hat{\Psi}\left(J^{\prime} \mathbf{1}^{\prime}, J^{\prime} \mathbf{b}^{\prime}\right)=\hat{\Psi}\left(J^{\prime} \overline{\mathbf{R}} \mathbf{1}^{\prime} \overline{\mathbf{R}}^{t}, J^{\prime} \overline{\mathbf{R}} \mathbf{b}^{\prime} \overline{\mathbf{R}}^{t}\right)
$$

for arbitrary proper-unimodular $\overline{\mathbf{R}}$. This representation problem has the same solution as that for $\hat{\Psi}(\mathbf{C}, \boldsymbol{\kappa})$ since the values of the functions $J(\cdot), \sigma(\cdot, \cdot)$ and $\kappa(\cdot)$, defined in (4.21), remain unaltered if $\Omega$ is replaced by $\omega^{\prime}$.

The more recent work of KRISHNASWAMY [10], which contains an extensive discussion and evaluation of the empirical literature on the phenomenology of biological bilayer response, is based on NAGHDI's [15] version of the KirchhoffLove theory together with what appears to be a hybrid constitutive assumption incorporating elements of the present models for films with and without a priori material symmetry. This study is perhaps the most comprehensive to date on the mechanics of biological fluid films and related structures. In it KRISHNASWAMY discusses unimodular transformations in relation to material symmetry but does not use them to obtain restrictions on response functions. His assumptions are apparently equivalent to those used to obtain (3.6), subject to the further requirement that the energy be a form-invariant function of the reference curvature with respect to affine coordinate transformations having arbitrary orthogonal coefficient matrices. In the notation of the present work this additional restriction is equivalent to the requirement that the energy be invariant under replacement of $\mathbf{B}$ with $\mathbf{R B R}{ }^{t}$, where $\mathbf{R}$ is an arbitrary orthogonal surface tensor. However, even if $\mathbf{B}$ is included explicitly as a parameter in the constitutive function, it does not seem possible to reconcile this assumption with the present theory unless restrictions are imposed on those surfaces that can be related by symmetry. To show this I note, with reference to 
(4.8), that Nanson's formula, which may be deduced directly from (4.1) and (4.6), yields

$$
\mathbf{N}=\left(A^{*} / A\right)^{1 / 2}(\operatorname{adj} \nabla \phi) \mathbf{N}^{*},
$$

where $\operatorname{adj}(\cdot)$ is the adjugate. From this and (4.8) it is evident that $\mathbf{B} \neq \mathbf{R} \mathbf{B}^{*} \mathbf{R}^{t}$ in general. Affine rotations are among those maps for which $A^{*}=A$ in accordance with the restriction to unimodular $\mathbf{R}$; for these it follows that $(\nabla \phi) \mathbf{N}^{*}=\mathbf{N}$, and, therefore, that $\mathbf{B}=\mathbf{R} \mathbf{B}^{*} \mathbf{R}^{t}$, but there is no reason to suppose that such maps furnish symmetry transformations for arbitrarily curved surfaces. Consistency with the present work is restored in the specialization to locally plane surfaces associated with the foregoing definition of fluidity, but Krishnaswamy's theory is then equivalent to the alternative model discussed in Section 3.

The propensity to identify the primitive concept of fluidity with isotropic response functions of $\mathbf{b}$ (cf. (3.6)) at the outset appears to have its roots in the work of some investigators [e.g., 7, 41, 42] who adapted the quadratic strain-energy function of Kirchhoff's classical (isotropic) linear plate bending theory to model biological fluid films with bending resistance. This practice remains widespread in the current literature on the phenomenology of surfactant systems $[1,2,43]$. There the energy is usually expressed as a linear combination of $K$ and $(2 H)^{2}$, which may be consistently confused with the isotropic invariants $\operatorname{det} \kappa$ and $(\operatorname{tr} \kappa)^{2}$ respectively in the applications envisaged for the original theory. However, when these variables are confused in the extension to finite deformations, such a formalism maintains its relevance to fluidity in the sense of NOLL only because the variables used are then unimodular invariants. The general issue is further complicated by the fact that solid-like response has been associated with films in highly condensed gel phases that exhibit long-range order as distinct from the high degree of in-plane mobility associated with fluid phases $[2,44]$. For these it is probable that a variant of the present symmetry theory based on orthogonal $\mathbf{R}$ is relevant, the transition to fluidity then being associated with response functions belonging to the class (3.6), or, equivalently, with a spontaneous enlargement of the symmetry group for the function (3.1), the latter being perhaps the more logically appealing framework. For bulk continua, the issue of symmetry as it relates to phase transition has been elucidated by RAJAGOPAL [45].

\section{Model equations}

Explicit component forms of the equilibrium equations for films belonging to the class (3.6) were derived by JENKINS [9] using a variational method. Formally, this class subsumes (4.21) and thus Jenkins' equations incorporate those for fluid films with symmetry. To show this I use (4.23), (4.24) and the local conservation of mass to write (4.22) in the form

$$
\Psi=G_{\lambda}^{\prime}\left(\rho, H, K ; \rho_{\lambda}(x), x\right) \doteq G_{\lambda}^{\prime \prime}(\rho, H, K ; x),
$$

where $\rho_{\lambda}$ is the density at particle $x$ in the reference configuration $\lambda$. From (4.22) it follows that the energy per unit mass at $x$ may be regarded as a function of $\rho, H$ and 
$K$ for any choice of reference configuration; in particular, relative to configuration $\mu$,

$$
\Psi=G_{\mu}^{\prime}\left(\rho, H, K ; \rho_{\mu}(x), x\right) \doteq G_{\mu}^{\prime \prime}(\rho, H, K ; x),
$$

where

$$
G_{\mu}^{\prime}\left(\rho, H, K ; \rho_{\mu}(x), x\right) \doteq G_{\lambda}^{\prime}\left(\rho, H, K ; \rho_{\mu}(x) D(x), x\right) .
$$

Thus, it may be viewed as a member of the class of functions $F$ in equation (3.6), in which parametric dependence on $x$ is permitted.

In the remainder of this section I obtain reduced equilibrium equations from the formulae of Sections 2 and 3. In light of Jenkins' analysis I will be brief. The reduction is facilitated by the fact that the divergence of the adjugate of the curvature tensor vanishes. To demonstrate this, let $\tilde{b}^{\alpha \beta}$ be the contravariant components of the adjugate of the symmetric surface tensor $\mathbf{b}$. Using (3.8) 2 , taking account of the skew-symmetry of $\varepsilon^{\alpha \beta}$, and regarding $b_{12}$ and $b_{21}$ as being independent, I obtain

$$
\tilde{b}^{\alpha \beta}=\frac{\partial K}{\partial b_{\alpha \beta}}=\varepsilon^{\alpha \lambda} \varepsilon^{\beta \gamma} b_{\lambda \gamma} .
$$

The Mainardi-Codazzi equations

$$
\varepsilon^{\alpha \lambda} b_{\gamma \lambda ; \alpha}=0
$$

the symmetry of $b_{\lambda \gamma}$, and the identity $\varepsilon_{; \gamma}^{\alpha \beta}=0$ then yield

$$
\tilde{b}_{; \alpha}^{\alpha \beta} \equiv 0
$$

as claimed. In view of this result it is advantageous to write the curvature in terms of its adjugate in expressions for the response functions. The appropriate formula follows from (6.4) and NAGHDI's treatise [15, equations (A.2.22) and (A.2.23)], or, directly from the Cayley-Hamilton theorem:

$$
b^{\alpha \beta}=2 H a^{\alpha \beta}-\tilde{b}^{\alpha \beta} .
$$

Here, $b^{\alpha \beta}$ are the contravariant components of $\mathbf{b}$.

To derive explicit forms of the response functions, it is necessary to evaluate the derivatives of $\rho, H$, and $K$ with respect to $a_{\alpha \beta}$ and $b_{\alpha \beta}$, regarded as independent matrices. Further, the off-diagonal entries of each of these matrices are regarded as being independent in accordance with (2.6). For example, if $a=\operatorname{det}\left(a_{\alpha \beta}\right)$, then

$$
\frac{\partial a}{\partial a_{\alpha \beta}}=a a^{\alpha \beta}
$$

and combining this with (2.7) gives

$$
\frac{\partial \rho}{\partial a_{\alpha \beta}}=-\frac{\rho}{2} a^{\alpha \beta} \text { and } \frac{\partial \rho}{\partial b_{\alpha \beta}}=0 .
$$


From (3.8) $)_{1}$, the derivatives of $H$ are

$$
\frac{\partial H}{\partial a_{\alpha \beta}}=-\frac{1}{2} b^{\alpha \beta} \text { and } \frac{\partial H}{\partial b_{\alpha \beta}}=\frac{1}{2} a^{\alpha \beta},
$$

the first of these having been obtained with the aid of the formula

$$
\frac{\partial a^{\lambda \mu}}{\partial a_{\alpha \beta}}=\varepsilon^{\lambda \alpha} \varepsilon^{\mu \beta}-a^{\lambda \mu} a^{\alpha \beta},
$$

which follows by differentiating $a^{\lambda \mu}=\varepsilon^{\lambda \alpha} \varepsilon^{\mu \beta} a_{\alpha \beta}$, with $a_{12}$ and $a_{21}$ regarded as being independent, and using (6.8). Finally, the derivatives of $K$ are given by (6.4) and

$$
\frac{\partial K}{\partial a_{\alpha \beta}}=-K a^{\alpha \beta},
$$

which follows from (4.23) and (6.8).

The response functions (2.6) are now easily shown to be

$$
\sigma^{\beta \alpha}=-\rho\left(\rho F_{\rho}+2 K F_{K}+2 H F_{H}\right) a^{\beta \alpha}+\rho F_{H} \tilde{b}^{\beta \alpha}
$$

and

$$
M^{\beta \alpha}=\rho\left(\frac{1}{2} F_{H} a^{\beta \alpha}+F_{K} \tilde{b}^{\beta \alpha}\right),
$$

where (6.7) has been used, the subscripts denote partial derivatives, and the symmetries of $a^{\alpha \beta}$ and $\tilde{b}^{\alpha \beta}$ have been used to condense the final results. Equations (6.13) may be combined with (2.5) and

$$
b_{\mu}^{\beta} \tilde{b}^{\mu \alpha}=K a^{\beta \alpha}
$$

to derive

$$
N^{\beta \alpha}=-\rho\left(\rho F_{\rho}+K F_{K}+H F_{H}\right) a^{\beta \alpha}+\frac{1}{2} \rho F_{H} \tilde{b}^{\beta \alpha},
$$

which is symmetric in the present theory.

Expressions for tractions and bending couples may be derived by substituting the foregoing formulae into (2.10) and (2.11).

I obtain explicit equilibrium equations by projecting (2.1) onto the tangent and normal spaces of $\omega$ at $x$ and using the Weingarten and Gauss equations

$$
\mathbf{n}_{, \alpha}=-b_{\alpha}^{\beta} \mathbf{a}_{\beta} \text { and } \mathbf{a}_{\alpha ; \beta}=b_{\alpha \beta} \mathbf{n} .
$$

Thus,

$$
N_{; \alpha}^{\beta \alpha}-S^{\alpha} b_{\alpha}^{\beta}=0, \quad S_{; \alpha}^{\alpha}+N^{\beta \alpha} b_{\beta \alpha}+p=0,
$$

where, from (2.8), (6.8) and (6.13) 2 ,

$$
-S^{\alpha}=\left(\frac{1}{2} \rho F_{H}\right)_{, \mu} a^{\alpha \mu}+\left(\rho F_{K}\right)_{, \mu} \tilde{b}^{\alpha \mu} .
$$


The foregoing formulae may be used to reduce $(6.17)_{2}$ to the final form

$$
p=\left(\frac{1}{2} \rho F_{H}\right)_{; \alpha \beta} a^{\alpha \beta}+\left(\rho F_{K}\right)_{; \alpha \beta} \tilde{b}^{\alpha \beta}+2 H \rho\left(\rho F_{\rho}+K F_{K}\right)+\rho\left(2 H^{2}-K\right) F_{H} .
$$

This coincides with its counterpart in JENKINs' work, which contains a minor typographical error.

The tangential equations $(6.17)_{1}$ reduce, after some effort, to

$$
a^{\beta \alpha}\left[\left(\rho^{2} F_{\rho}\right)_{, \alpha}+\rho\left(F_{K} K_{, \alpha}+F_{H} H_{, \alpha}\right)\right]=0,
$$

which is equivalent to the vanishing of the expression in brackets by virtue of the definiteness of the metric. In the classical theory of capillarity in which $F_{K}$ and $F_{H}$ vanish identically, this yields the well-known result that the surface pressure $\rho^{2} F_{\rho}$ is uniform. In the general case, (6.20) is equivalent to

$$
\left(\rho F_{\rho}\right)_{, \alpha}+F_{\rho} \rho_{, \alpha}+F_{K} K_{, \alpha}+F_{H} H_{, \alpha}=0,
$$

which in turn may be written

$$
\left(\rho F_{\rho}+F\right)_{, \alpha}=\partial F / \partial \theta^{\alpha},
$$

where the right-hand side is associated with the explicit dependence of $F$ on $x$. This result was obtained by JENKINS for homogeneous films with no such dependence, and in that context furnishes an integral of the equations which generates a oneparameter relationship among $\rho, H$ and $K$. For films with material symmetry, (6.1)(6.3) imply that homogeneity, if it exists, is a property of the reference configuration. Homogeneity is not preserved under transformations from one to another, unless they are density preserving or $D(x)$ is constant.

In the general case, $(6.22)$ is integrable in any simply-connected region of the surface if and only if $e^{\alpha \beta} I_{\alpha, \beta}=0$, where $e^{\alpha \beta}$ is the unit alternator and $I_{\alpha}=\partial F / \partial \theta^{\alpha}$ is the inhomogeneity. Exceptionally, (6.22) is satisfied identically by solutions of the classical theory without restrictions on the inhomogeneity. In any event, (6.19) and the integrability condition for (6.22) furnish an underdetermined system for the components of the three-vector field $\mathbf{r}\left(\theta^{\alpha}\right)$. Thus, as in the classical theories of capillary and bulk-fluid equilibria, the equilibrium equations do not suffice to determine the locations of material particles. This is in accord with the intuitive idea of fluidity.

Finally, I note that all the equations of this section remain valid in the presence of the two-dimensional incompressibility constraint provided $F(\rho, H, K)$ is replaced by

$$
F=\bar{F}(H, K)-\gamma / \rho,
$$

where $\bar{F}$ is a constitutive function and $\gamma=\rho^{2} F_{\rho}$ is the constitutively-indeterminate surface pressure (cf. (2.8)). 


\section{Energy minimizers}

I obtain the quasiconvexity condition and related algebraic inequalities associated with necessary conditions for energy minimizers. Although the minimum energy test is inconclusive with respect to the dynamical stability of equilibria, it nevertheless furnishes a formal necessary condition for asymptotic stability if the associated dynamics are strictly dissipative [46, 47]. Granted this it is then also necessary that stable equilibria furnish non-negative values of the second variation of the energy. Thus, as in Section 2, let superposed dots denote derivatives with respect to a parameter $\varepsilon$ that labels configurations, evaluated at the equilibrium state $\varepsilon=0$. The second variation of the energy functional (2.16) may then be written

$$
\ddot{E}=\sum_{i} \int_{V_{i}}\left(\ddot{U}+\varrho_{0} g \mathbf{k} \cdot \ddot{\chi}\right) \mathrm{d} V+\sum_{j} \int_{\Omega_{j}} \ddot{W} \mathrm{~d} A,
$$

where $V_{i}$ and $\Omega_{j}$ are fixed reference configurations of the bulk fluids and the films, and $U=\varrho_{0} \Phi$ and $W=\rho_{0} \Psi$ are the bulk and film energies per unit reference volume and area respectively. The first of these is a function of the bulk fluid deformation function $\chi(X ; \varepsilon)$, as discussed in Section 2; the second, a function of the metric and curvature induced by the parametrization $\mathbf{r}\left(\theta^{\alpha} ; \varepsilon\right)$.

The second variation at an equilibrium state is a homogeneous quadratic functional of the first-order derivatives $\dot{\chi}$ and $\dot{\mathbf{r}}$. Using this state as reference, I write

$$
\ddot{E}=\sum_{i} \int_{v_{i}} A(\operatorname{grad} \mathbf{u}) \mathrm{d} v+\sum_{j} \int_{\omega_{j}} B\left(\dot{a}_{\alpha \beta}, \dot{b}_{\alpha \beta}\right) \mathrm{d} a,
$$

where $\mathbf{u}(\mathbf{y})=\dot{\chi}$,

$$
\dot{a}_{\alpha \beta}=\mathbf{a}_{\alpha} \cdot \mathbf{v}_{, \beta}+\mathbf{a}_{\beta} \cdot \mathbf{v}_{, \alpha}, \quad \dot{b}_{\alpha \beta}=\mathbf{n} \cdot \mathbf{v}_{; \alpha \beta}
$$

where $\mathbf{v}\left(\theta^{\alpha}\right)=\dot{\mathbf{r}}$, and the covariant derivative is based on the metric induced by $\mathbf{r}\left(\theta^{\alpha}\right)$ at $\varepsilon=0$ [24]. Further, $A(\cdot)$ and $B(\cdot, \cdot)$ are homogeneous quadratic functions involving the second derivatives of $U$ and $W$ with respect to their arguments. Equation (7.2) applies whether or not the two- or three-dimensional incompressibility constraints are operative [25, 26].

To obtain the quasiconvexity condition I consider variations of the form

$$
\mathbf{u}(\mathbf{y})=\operatorname{curl} \mathbf{w}(\mathbf{y}) ; \quad \mathbf{w}(\mathbf{y})=\delta^{3} \hat{\mathbf{w}}(\mathbf{z}(\mathbf{y})), \quad \text { and } \quad \mathbf{v}\left(\theta^{\alpha}\right)=\delta^{2} \hat{\mathbf{v}}\left(\boldsymbol{\eta}\left(\theta^{\alpha}\right)\right),
$$

where

$$
\mathbf{z}(\mathbf{y})=\delta^{-1}\left(\mathbf{y}-\mathbf{r}_{0}\right) \quad \text { and } \quad \boldsymbol{\eta}\left(\theta^{\alpha}\right)=\mathbf{z}\left(\mathbf{r}\left(\theta^{\alpha}\right)\right) .
$$

Here, $\delta$ is a positive number and the functions $\hat{\mathbf{w}}(\cdot), \hat{\mathbf{v}}(\cdot)$ have compact support in a three-dimensional region $D$ containing a point $\mathbf{r}_{0}$ on one of the films, $\omega_{0}$ say. I assume $\delta$ to be small enough that the intersection of $D$ with any other film is empty. 
Since $\mathbf{u}(\mathbf{y})$ is solenoidal, it automatically satisfies the variational form of the incompressibility constraint in the bulk fluid. Further, the requirement that there be no flux of fluid across the film (Section 2) implies that

$$
\left.\mathbf{u} \cdot \mathbf{n}\right|_{\omega_{0}}=\mathbf{v} \cdot \mathbf{n} \doteq v\left(\theta^{\alpha}\right),
$$

where $\mathbf{n}$ is the local orientation field on $\omega_{0}$. If the film is incompressible in the sense that local surface area is preserved, then the surface divergence of $\mathbf{v}$ vanishes [23], and the representation $\mathbf{v}=v^{\alpha} \mathbf{a}_{\alpha}+v \mathbf{n}$ yields

$$
v_{; \alpha}^{\alpha}=2 H v
$$

where $H$ is the mean curvature of $\omega_{0}$.

Local normal coordinates [15] may be used with $(7.4)_{1}$ to reduce (7.6) to the form

$$
\varepsilon^{\alpha \beta} w_{\beta ; \alpha}=v,
$$

where $w_{\beta}\left(\theta^{\alpha}\right)=\left.\mathbf{a}_{\beta} \cdot \mathbf{w}\right|_{\omega_{0}}$ and $v$ vanishes on the curve $c=\partial D \cap \omega_{0}$. With $v$ prescribed, the existence of a covariant vector field satisfying this equation may be proved by writing $\varepsilon^{\alpha \beta} w_{\beta}=a^{\alpha \beta} \phi_{, \beta}$, which has a unique solution $w_{\alpha}$. Then, for a given parametrization of $\omega_{0}$, (7.8) reduces to a second-order linear elliptic equation for $\phi$. For sufficiently smooth Dirichlet data on $c$ the existence of a unique solution follows from [48, Theorem 21(I)]. Unfortunately such a scheme does not yield the existence of $v^{\alpha}$ satisfying (7.7) for incompressible films. For example, setting $v^{\alpha}=$ $a^{\alpha \beta} \phi_{, \beta}$, I again obtain a linear elliptic equation for $\phi$, but the additional requirement that $v^{\alpha}$ vanish on $c$ entails the simultaneous specification of homogeneous Dirichlet and Neumann data for $\phi$. Exceptionally, if $\omega_{0}$ is a minimal surface $(H \equiv 0)$ in a neighborhood of $\mathbf{r}_{0}$, then $v^{\alpha}$ may be any divergence-free vector field that vanishes on $c$. Alternatively, one may specify a vector field $v^{\alpha}$ which vanishes together with its divergence on $c$ and use (7.7) to calculate $v$ at points where $H \neq 0$, but in the absence of detailed information about the surface $\omega_{0}$ it may not be feasible to ensure that $\mathbf{v}$ then possesses the properties required to generate the Legendre-Hadamard condition from the quasiconvexity inequality. This is due to the fact that it is the component of $\mathbf{v}$ normal to the tangent plane at $\mathbf{r}_{0}$, rather than $v$, that is relevant, as shown below.

Let $\nabla(\cdot)$ and $\nabla \times(\cdot)$ denote the gradient and curl with respect to $\mathbf{z}$. Then,

$$
\begin{aligned}
\mathbf{u}(\mathbf{y}) & =\delta^{2} \nabla \times \hat{\mathbf{w}}(\mathbf{z}), \quad \operatorname{grad} \mathbf{u}(\mathbf{y})=\delta \nabla(\nabla \times \hat{\mathbf{w}}), \\
\mathbf{v}_{, \alpha} & =\delta(\nabla \hat{\mathbf{v}}) \mathbf{a}_{\alpha} \quad \text { and } \quad \mathbf{v}_{, \alpha \beta}=(\nabla \nabla \hat{\mathbf{v}})\left[\mathbf{a}_{\alpha} \otimes \mathbf{a}_{\beta}\right]+\delta(\nabla \hat{\mathbf{v}}) \mathbf{a}_{\alpha, \beta} .
\end{aligned}
$$

Now, let $u^{\alpha}$ be smooth extensions of the coordinates $\theta^{\alpha}$ onto the plane tangent to $\omega_{0}$ at $\mathbf{r}_{0}$. I take these to be affine coordinates such that $\partial \boldsymbol{\eta} / \partial u^{\alpha}=\stackrel{\mathbf{a}}{\alpha}_{\alpha}$, the superposed circle identifying the values of functions at $\mathbf{r}_{0}$. Thus, $\partial^{2} \boldsymbol{\eta} / \partial u^{\alpha} \partial u^{\beta}=\mathbf{0}$, and it follows that

$$
(\nabla \nabla \hat{\mathbf{v}})\left[\stackrel{\mathbf{a}}{\alpha}_{\alpha} \otimes \mathbf{a}_{\beta}\right]=\hat{\mathbf{v}}_{, \alpha \beta},
$$

where, here and henceforth, commas denote derivatives with respect to $u^{\alpha}$. 
I change variables in accordance with (7.5) 1 and use (7.3), (7.9) and (7.10). Holding $D$ fixed, I divide the second-variation inequality by $\delta^{2}$ and pass to the limit to obtain the quasiconvexity condition for the film:

$$
B^{\alpha \beta \lambda \mu} \int_{\omega^{*}} w_{, \alpha \beta} w_{, \lambda \mu} \mathrm{d} a \geqq 0,
$$

where $w=\grave{\mathbf{n}} \cdot \hat{\mathbf{v}}, \omega^{*}$ is the intersection of $D$ with the tangent plane of $\omega_{0}$ at $\mathbf{r}_{0}$, and

$$
B^{\alpha \beta \lambda \mu}=\rho \frac{\partial^{2} \Psi}{\partial b_{\alpha \beta} \partial b_{\lambda \mu}}
$$

is evaluated at $\mathbf{r}_{0}$ in the configuration $\omega_{0}$. The symmetries inherent in this tensor ensure that (7.11) is equivalent to the inequality obtained by replacing the integrand with $w_{, \alpha \beta} \bar{w}_{, \lambda \mu}$, where $w$ is now complex-valued and $\bar{w}$ is its conjugate. The resulting inequality is in standard form for generating the relevant Legendre-Hadamard condition (e.g., [49, pp. 229-231]). For $w$, I choose

$$
w\left(u^{\alpha}\right)=a \phi\left(u^{\beta}\right) \exp \left[i \tau\left(\varsigma_{\alpha} u^{\alpha}\right)\right],
$$

where $a, \tau(>0)$ and $\zeta_{\alpha}$ are real constants and $\phi$ is a real $C^{\infty}$ function supported in $\omega^{*}$. Then,

$$
w_{, \alpha \beta} \bar{w}_{, \lambda \mu}=a^{2} \phi^{2} \varsigma_{\alpha} \varsigma_{\beta} \varsigma_{\lambda} \varsigma_{\mu}+O\left(\tau^{-1}\right),
$$

and passing to the limit $\tau \rightarrow \infty$ in (7.11) yields the necessary condition

$$
B^{\alpha \beta \lambda \mu} \varsigma_{\alpha} \varsigma_{\beta} \varsigma_{\lambda} \varsigma_{\mu} \geqq 0
$$

for all $\varsigma_{\alpha}$. I conjecture that this condition is also necessary in the presence of the two-dimensional incompressibility constraint. A similar inequality was obtained by HILGERS \& PIPKIN [21] for elastic plates isolated from other media.

For fluid films the associated restriction on minimizing states follows by using (3.6), (6.4) and (6.10) to reduce (7.15) to the form

$$
\frac{1}{4} F_{H H}+2 x F_{H K}+x^{2} F_{K K} \geqq 0 \text { where } x=\tilde{b}^{\alpha \beta} \varsigma_{\alpha} \varsigma_{\beta} .
$$

In this expression the first-order derivative of $F$ with respect to $H$ does not appear because $H$ is a linear function of the matrix $b_{\alpha \beta}$; the first-order derivative with respect to $K$ does not appear because the second derivatives of $K$ with respect to this matrix involve the permutation tensor density in such a way as to make no contribution to (7.15).

For incompressible films (cf. (6.23)), several forms of the function $\bar{F}(H, K)$ compatible with (7.16) have been proposed. Among them, HeLFRICH's function [7]

$$
\rho \bar{F}=\alpha\left(H-H_{0}\right)^{2}+\beta K, \quad \text { with } \alpha>0,
$$

has been the most widely applied and studied. The spontaneous curvature $H_{0}$ is a parameter introduced to render the energy an non-even function of the curvature tensor. The resulting formulation is thought to furnish an appropriate model for 
monolayer films such as those associated with oil-in-water or water-in-oil emulsions [1]. Bilayer response is recovered by setting $H_{0}=0$. Some writers model morphological phase transformations by allowing $H_{0}$ to depend on amphiphile concentration, which in turn is the principal factor influencing local film chemistry [50].

Existence theory for the local equilibrium equations based on Helfrich's function and related functions has been discussed by NiTsche [51]. However, from the viewpoint of variational theory, Helfrich's model is deficient in the sense that the energy of a given film is generally not bounded below. This is easily seen with reference to compact orientable films by applying the Gauss-Bonnet formula

$$
\int_{\omega} K \mathrm{~d} a=4 \pi(1-g)
$$

where $g$ is the genus [52]. For $\beta>0$, the second term in (7.17) contributes a term to the total film energy that decreases without bound as $g$ increases. The same term contributes only a fixed constant to the energy in the presence of the topological constraints imposed by some writers [7,11].

A simple alternative model for bilayers, as yet unexplored, may be based on the assumption that $\bar{F}$ is a function of $K$ depending parametrically on temperature and amphiphile concentration. At fixed concentration and temperature, the graph of such a function might exhibit local minima at $K>0,=0$, and $<0$. These correspond to points of convexity of the energy in accordance with (7.16). The first alternative promotes the formation of spherical vesicles interspersed in the bulk fluid; the second, developable surfaces, including the cylindrical and lamellar phases; and the third, the bicontinuous phases associated with compact orientable surfaces having large genus [3,4]. In the latter case the minimizing value of $K$ cannot be achieved at all points of the film as there are no surfaces in 3-space with constant negative Gaussian curvature [53]. Nevertheless it is appropriate to conjecture that configurations with high genus are promoted by a sufficiently deep and wide energy-well spanning an interval of the domain of $\bar{F}$ in which $K<0$. The structure of the energy-wells might depend on concentration and temperature in such a way as to favor some of these structures over others in accordance with the observed phase behavior of the particular system at hand.

\section{References}

1. Kellay, H., Binks, B. P., Hendrikx, Y., Lee, L. T. \& Meunier, J., Properties of surfactant monolayers in relation to microemulsion phase behaviour. Advances in Colloid and Interface Science, 49, 85-112 (1994).

2. Gelbart, W. M., Ben-Shaul, A. \& Roux, D. (editors), Micelles, Membranes, Microemulsions, and Monolayers. Springer Series on Partially Ordered Systems. SpringerVerlag, New York, 1994.

3. SCRIVEN, L. E., Equilibrium bicontinuous structure. Nature, 263, 123-125 (1976).

4. Scriven, L. E., Equilibrium bicontinuous structures. In: Micellization, Solubization, and Microemulsions, vol. 2, K.L. Mittal (ed.), Plenum Press, New York, pp. 877-893, 1977. 
5. Evans, E. A. \& Skalak, R., Mechanics and thermodynamics of biomembranes. CRC Press, Boca Raton, FL., 1980.

6. Collings, P. J., Liquid crystals: nature's delicate phase of matter. Princeton University Press, Princeton, N.J., 1990.

7. Helfrich, W., Elastic properties of lipid bilayers: theory and possible experiments. $Z$. Naturforsch.,28c, 693-703 (1973).

8. ERICKSEN, J.L., Theory of Cosserat surfaces and its applications to shells, interfaces and cell membranes. In: Proc. Int. Symp. on Recent Developments in the Theory and Application of Generalized and Oriented Media (P. G. Glockner, M. Epstein \& D. J. Malcolm, eds.), pp. 27-39, Calgary, 1979.

9. Jenkins, J. T., The equations of mechanical equilibrium of a model membrane. SIAM J. Appl. Math., 32, 755-764 (1977).

10. Krishnaswamy, S., A Cosserat-type model for the red blood cell wall. Internat. $J$. Engng. Sci., 34, 873-899 (1996).

11. Jenkins, J. T., Static equilibrium configurations of a model red blood cell. J. Math. Biology, 4, 149-169 (1977).

12. Charvolin, J. \& SAdOc, J.-F., Geometrical foundation of mesomorphic polymorphism. In [2] pp. 218-249.

13. NolL, W., A mathematical theory of the mechanical behavior of continuous media. Archive for Rational Mechanics and Analysis, 2, 197-226 (1958).

14. Murdoch, A. I. \& Cohen, C., Symmetry considerations for material surfaces. Archive for Rational Mechanics and Analysis, 72, 61-89 (1979).

15. Naghd, P. M., Theory of shells and plates. In: Handbuch der Physik, Vol. VI/2 (C. Truesdell, ed.), Springer-Verlag, Berlin, pp.425-640, 1972.

16. KirchноғF, G., Über das Gleichgewicht und die Bewegung einer elastischen Scheibe. J. Reine u. Angew. Math., 40, 51-88 (1850).

17. Sanders, J. L., Nonlinear theories for thin shells. Quart. Appl. Math., 21, 21-36 (1963).

18. Pietraszkiewicz, W., Geometrically nonlinear theories of thin elastic shells. Advances in Mechanics, 12, 52-130 (1989).

19. Antman, S. S., Nonlinear problems of elasticity. Springer-Verlag, New York, 1995.

20. Steigmann, D. J., On the relationship between the Cosserat and Kirchhoff-Love theories of elastic shells. Mathematics and Mechanics of Solids, 4, 275-288 (1999).

21. Hilgers, M. G. \& PiPKIN, A. C., Energy minimizing deformations of elastic sheets with bending stiffness. J. Elasticity, 31, 125-139 (1993).

22. NAGHDI, P. M., Finite deformation of elastic rods and shells. In: Proc. IUTAM Symposium on Finite Elasticity (CARLSON, D.E. \& ShIELD, R.T., eds.), Martinus Nijhoff, The Hague, pp. 47-103 (1980).

23. Steigmann, D. J. \& Li, D., Energy minimizing states of capillary systems with bulk, surface, and line phases. IMA J. Appl. Mathematics, 55, 1-17 (1995).

24. Steigmann, D .J. \& Ogden, R. W., Elastic surface-substrate interactions. Proc. R. Soc. Lond., A 455, 437-474 (1999).

25. LEE, S. J. \& SHIELD, R. T., Variational principles in finite elastostatics. ZAMP, 31, 437453 (1980).

26. Fosdick, R. L. \& MacSithigh, G. P., Minimization in incompressible nonlinear elasticity theory. J. Elasticity, 16, 267-301 (1986).

27. BALL, J. M., On the calculus of variations and sequentially weakly continuous maps. In: Lecture Notes in Mathematics, Vol. 564 (Ordinary and Partial Differential Equations), Springer-Verlag, Berlin, 1976.

28. Collected Papers of R. S. Rivlin (Barenblatt, G. I. \& Joseph, D. D., eds.), Vols. I, II. Springer-Verlag, New York, 1997.

29. ERICKSEN, J. L., Equilibrium theory of liquid crystals. In: Advances in Liquid Crystals, (Brown, G. H., ed.), Academic Press, New York, pp. 233-298, 1976.

30. Landau, L. D. \& Lifschitz, E.M., Theory of elasticity, 3rd edn.. Pergamon Press, Oxford, 1986

31. Moeckel, G. P., Thermodynamics of an interface. Archive for Rational Mechanics and Analysis, 20, 255-280 (1975). 
32. Zheng, Q. S., Two-dimensional tensor function representation for all kinds of material symmetry. Proc. R. Soc. Lond., A443, 127-138 (1993).

33. Blinowski, A., On curvature dependent surface tension. Archiwum Mechaniki Stosowanej, 23, 213-222 (1971).

34. FAetti, S. \& Virga, E. P., On a curvature surface energy for nematic liquid crystals. Archive for Rational Mechanics and Analysis, 140, 31-52 (1997).

35. Jenkins, J. T. \& Barrat, P. J., Interfacial effects in the static theory of nematic liquid crystals. Quart. J. Mech. Appl. Math., 27, 111-127 (1974).

36. Ericksen, J. L., Liquid crystals and Cosserat surfaces. Quart. J. Mech. Appl. Math., 27, 213-219 (1974)

37. ERICKSEN, J. L., Symmetry transformations for thin elastic shells. Archive for Rational Mechanics and Analysis, 47, 1-14 (1972).

38. Carroll, M. M. \& NAGHdi, P. M., The influence of reference geometry on the response of elastic shells. Archive for Rational Mechanics and Analysis, 48, 302-318 (1972).

39. Cohen, H. \& WANG, C.-C., On the response and symmetry of elastic and hyperelastic membrane points. Archive for Rational Mechanics and Analysis, 85, 343-379 (1984).

40. Rivlin, R. S. \& Sмith, G. F., Comments on the paper: 'On the derivation of the constitutive equation of the simple fluid from that of the simple material' by R. R. Huilgol, Rheologica Acta, 28, 246-252 (1989), reprinted in [28].

41. Fung, Y.C., Theoretical considerations of the elasticity of red blood cells and small blood vessels. Proc. Fed. Am. Soc. Exp. Biol., 25, 1761 (1966).

42. Kléman, M., Remarks on a possible elasticity of membranes and lamellar media: disordered layers. Proc. R. Soc. Lond., A347, 387-404 (1976).

43. Charvolin, J. \& SAdOc, J.-F., Ordered bicontinuous films of amphiphiles and biological membranes. Phil. Trans. R. Soc. London, A354, 2173-2192 (1996).

44. BIRDI, K. S., Lipid and biopolymer monolayers at liquid interfaces. Plenum Press, New York, 1989.

45. Rajagopal, K. R. On constitutive relations and material modeling. Preprint.

46. ERICKSEn, J. L., A thermo-kinetic view of elastic stability theory. Internat. J. Solids and Structures, 2, 573-580 (1966).

47. Koiter, W. T., A basic open problem in the theory of elastic stability. In: Proc. IUTAM/IMU Symp. on Applications of Methods of Functional Analysis to Problems in Mechanics (Germain, P. \& Nayroles, B., eds.), Springer-Verlag, Berlin, pp. 366-373, 1976.

48. Miranda, C., Partial differential equations of elliptic type. Springer-Verlag, Berlin, 1970.

49. Giaquinta, M. \& Hildebrandt, S., Calculus of variations I. Springer-Verlag, Berlin, 1996.

50. Safran, S. A., Fluctuating interfaces and the structure of microemulsions. In: [2] pp. 427-484.

51. Nitsche, J.C. C., Periodic surfaces that are extremal for energy functionals containing curvature functions. In: Statistical Thermodynamics and Differential Geometry of Microstructured Materials, (DAvis, H.T. \& Nitsche, J. C. C., eds.), Springer-Verlag, New York, pp. 69-98, 1993.

52. Hopf, H., Differential geometry in the large. Lecture Notes in Mathematics, Vol. 1000 (Lectures delivered at New York University, 1946, and Stanford University, 1956), Springer-Verlag, 1980.

53. Stoker, J. J., Differential geometry. Wiley-Interscience, New York, 1969.

Department of Mechanical Engineering

University of California

Berkeley, CA 94720

(Accepted February 22, 1999) 